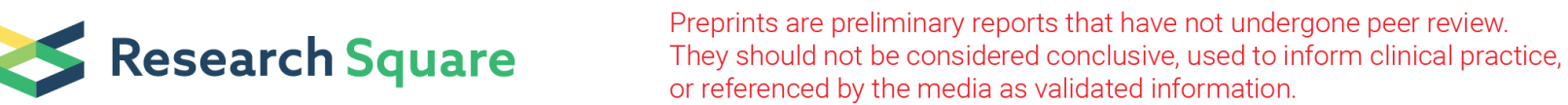

\section{Nurses suffered with more psychological symptoms when fighting against novel coronavirus pneumonia (COVID-19): a longitudinal observational survey of medical staff}

\section{You-Ping Zhang}

Huazhong University of Science and Technology Tongji Medical College https://orcid.org/0000-00032654-6418

Hai-Tao Yuan

Dongxi Lake District People's Hospital

\section{Ya-Qin Song}

Huazhong University of Science and Technology Tongji Medical College

Jing Jia

Tongji Hospital of Tongji Medical College of Huazhong University of Science and Technology

\section{Wei Mi}

Dongxi Lake District People's Hospital

\section{Yan-Lin Wang}

Dongxi Lake District People's Hospital

\section{Jia Wang}

Dongxi Lake District People's Hospital

\section{Da-Yong Li}

Tongji Hospital of Tongji Medical College of Huazhong University of Science and Technology

Wei Zhu ( $\sim$ tjjzkzw512@163.com )

\section{Primary research}

Keywords: community hospital, medical staff, novel coronavirus pneumonia, psychological status, symptom checklist 90

Posted Date: June 16th, 2020

DOl: https://doi.org/10.21203/rs.3.rs-35668/v1

License: (c) (i) This work is licensed under a Creative Commons Attribution 4.0 International License. Read Full License 


\section{Abstract \\ Objective.}

Our objective is to investigate and analyze the psychological status of medical staff in a designated community hospital for COVID-19.

\section{Methods.}

We conducted a survey on medical staff in a designated community hospital for COVID-19 among the during-pandemic group $(n=120)$ and the after-pandemic group $(n=34)$. The symptom checklist 90 (SCL90) questionnaire was used as a self-report instrument for the measurement of psychopathological complaints.

\section{Results.}

The during-pandemic group consisted of 120 individuals, including doctors $(n=36)$, nurses $(n=69)$ and technicians $(n=15)$. The SCL-90 sum scores showed no difference among doctors, nurses, and technicians $(P>0.05)$, but the somatization (SOM) item-scores of nurses were significantly higher than those of doctors and technicians (all $\mathrm{P}<0.05$ ). Meanwhile, the paranoid ideation $(P A R)$ item-scores of nurses were significantly higher than those of doctors $(P<0.05)$. The after-pandemic group consisted only of nurses $(n=34)$. The score of each dimension of SCL-90 in nurses after the pandemic were relatively lower than that in nurses during the pandemic.

\section{Conclusions.}

Our study showed that nurses suffered with more psychological symptoms when fighting against COVID19. The emergence of COVID-19 was the main factor leading to psychological problems of nurses.

\section{Introduction}

Since December 2019, the first COVID-19 case has been found in Wuhan, Hubei province, the virus has caused at least 80000 cases in mainland China. The tremendous numbers of patients quickly overwhelmed the health and public health services in Wuhan [1], and the community hospital is the first line of defense and plays an important role in the treatment of COVID-19. The COVID-19 brought to medical staff not only the higher risk of infection, but also unbearable psychological pressure.

In fact, a series of studies reported mental symptoms existed among healthcare professionals [2-4], especially for those medical workers who worked in an extraordinary situation and were prone to various psychological and mental problems because of exhaustion, frustration, discrimination, and so on. Health 
professionals who worked in severe acute respiratory syndrome (SARS) units and hospitals during the SARS outbreak also presented depression, anxiety, fear, and frustration [5]. At present, numerous researchers revealed that mental health care was urgently needed in face of COVID-19 pandemic [6-8]. Unhealthy mental states can lead to a decrease in work efficiency and even affect the health of medical workers themselves. Therefore, a survey on the psychological state of medical workers is of vital importance and urgently needed. However, few studies have explored the mental health and its related factors of medical workers in community hospitals during the COVID - 19 pandemic.

In order to evaluate the psychological state of medical staff in a community hospital, we adopt the symptom checklist 90 (SCL-90), an international, self-report questionnaire, to investigate psychological factors [9-11] and conducted a survey both at the height of COVID-19 pandemic and after the pandemic. We intended to find the differences among medical staff with SCL-90 questionnaire in a designated community hospital.

\section{Materials And Methods}

\section{Participants}

The participants are made up of two groups. During-pandemic group $(n=136)$ were medical staff fighting against COVID-19 at the height of the outbreak at a community hospital, and after-pandemic group $(\mathrm{n}=$ 34) were nurses at the same community hospital returning from the first line after all COVID-19 patients there were cleared and the community hospital reopened to the public. We conducted the survey of during-pandemic group on February 17th 2020 and conducted that of after-pandemic group on May 17th, 2020. In the during-pandemic group, participants consisted of doctors, nurses and technicians. Each doctor and nurse had a four-hour shift every day in the contaminated area. Technicians worked in semicontaminated area for six hours every day. In the after-pandemic group, all nurses had an eight-hour shift every day. Junior college or below was defined as a low level of education, bachelor's degree as a medium level of education and master's degree or above as a high level of education. The SCL-90 questionnaire was used as a self-report instrument for the measurement of psychopathological complaints. All participants gave informed consent. Briefly, all participants were trained by an investigator and then required to rate how much they had suffered from each of the listed symptoms within the last 7 days. Participation was anonymous, as there was no name or other identification code on the booklet. The medical ethics committee of Dongxi Lake district people's hospital approved the research protocol.

\section{Instruments}

The SCL-90 questionnaire comprises 90 items that assess psychopathological or somatic disturbances on a 5 -point scale ranging from 1 (absence of symptom) to 5 (maximum disturbance). The questionnaire contains nine primary symptom dimensions: somatization (SOM), obsession-compulsion (O-C), interpersonal sensitivity (I-S), depression (DEP), anxiety (ANX), hostility (HOS), phobic anxiety (PHOB), 
paranoid ideation (PAR), and psychoticism (PSY). Each of the nine symptom dimensions is assessed with 6 to13 items [12]. The score on each dimension represents the mean score of all items of the dimension and directly reflects the severity of the mental health problem. Subscale scores $\geq 2$ were suggestive of potential mental health issues. The global severity index (GSI) is a mean score of all 90 items.

\section{Statistical analysis}

Data manual analysis was performed independently by two investigators unaware of the status of individuals. The data were presented as mean \pm SD unless otherwise specified. Statistical analysis included Fisher exact probability test for categorical variables and ANOVA or T test for continuous variables. All statistical calculations were performed using SPSS 17.0 (IBM Corporation, Armonk, NY, USA). $P$ values $<0.05$ were considered significant and all reported $P$ values were two sided.

\section{Results}

\section{Participants characteristics}

In the during-pandemic group, 136 individuals had enrolled. Among them, 16 people failed to complete the self-report measure and were excluded. The definitive analysis was performed on the remaining 120 individuals, including doctors $(n=36)$, nurses $(n=69)$ and technicians $(n=15)$. The mean age of the during-pandemic group was $31.89 \pm 7.48$ years, and doctors were older than nurses and technicians (37.5 $\pm 7.97, v s$. $29.67 \pm 5.64$ and $28.67 \pm 6.76$, all $\mathrm{P}<0.05$ ). The during-pandemic group comprised 37 men $(30.8 \%)$ and 83 women $(69.2 \%)$, and the male ratio in doctors and technicians were higher than that in nurses, separately $(69.4 \%$ and $66.7 \%$ vs. $12.9 \%$, all $\mathrm{P}<0.01)$. The proportions of nurses in three different education levels were significantly different from that of doctors, and the proportions of nurses in low and medium levels of education were significantly different from that of technicians (all P< 0.05 ). (Table 1)

In the after-pandemic group, 34 nurses had enrolled and all of them completed the self-report measure. The mean age of the nurses was $27.74 \pm 4.65$ years and there were no differences in gender, age or education level between two groups of nurses. (Table 1 )

\section{SCL-90 scores of three types of professionals during the pandemic}

The SCL-90 sum scores showed no difference among doctors, nurses, and technicians $(P>0.05)$, but the SOM item-scores of nurses $(1.51 \pm 0.54)$ were significantly higher than those of doctors $(1.28 \pm 0.31)$ and technicians $(1.19 \pm 0.27)$ (all $P<0.05)$. Meanwhile, PAR item-scores of nurses $(1.23 \pm 0.41)$ were significantly higher than those of doctors $(1.09 \pm 0.16)(P<0.05)$. (Table 2, Fig. 1-a)

SOM and PAR item-scores of three types of professionals during the pandemic 
The SOM and PAR item-scores of three types of professionals were showed in Table 3. Of the twelve items of the SOM item-scores, the scores of item 27, 40, 42, 48, 49 and 52 were significantly higher in nurses than those in doctors (all $P<0.05$ ), and the scores of item 27, 40, 42 and 52 were significantly higher in nurses than those in technicians (all $P<0.05$ ). Of the six items of the PAR item-scores, the scores of item 18 and 76 were significantly higher in nurses than those in doctors (all $P<0.05$ ). (Table 3 )

\section{Influence of gender, profession and education level on the SCL-90 score during the pandemic}

The SCL-90 scores of females were relatively higher than those of males, and significant difference were existed in SOM item-scores, ANX item-scores and PAR item-scores (all P< 0.05, Fig. 1-b). The SCL-90 scores of nurses were relatively higher than non-nurses, and significant difference were existed in SOM item-scores, O-C item-scores, ANX item-scores, GSI item-scores and PAR item-scores (all $P<0.05$, Fig. 1-c). However, the SCL-90 scores in three different education levels showed no differences (all P>0.05, Fig. 1d).

\section{The SCL-90 scores of nurses during the pandemic and after the pandemic}

The SCL-90 sum scores of nurses during the pandemic and after the pandemic were $125.62 \pm 44.08$ and $107.24 \pm 15.74$, respectively. The score of each dimension of SCL-90 in nurses during the pandemic were relatively higher than that in nurses after the pandemic. In nurses during the pandemic, the highest score and the lowest score of dimensions were $0-C(1.64 \pm 0.66)$ and PSY $(1.20 \pm 0.36)$, respectively. Similarly, in nurses after the pandemic, the highest score and the lowest score of dimensions were O-C $(1.44 \pm 0.33)$ and PSY (1.06 \pm 0.16$)$, respectively. In fact, except for HOS and PAR, item-scores of SOM, O-C, I-S, DEP, $A N X, P H O B, P S Y$ and GSI in nurses during the pandemic were significantly higher than that in nurses after the pandemic (all $P<0.05$ ). (Fig. 2)

\section{Discussion}

Currently, the number of COVID-19 patients is increasing rapidly, which is a serious threat to human's health $[13,14]$. Community hospitals play an important role and are the main battlefields to fight against COVID-19. The COVID-19 brought to medical workers in community hospitals not only the fear of infection, but also unbearable psychological pressure [5], which in turn affects the quality of medical care and health of medical workers. One meta-analysis once revealed that Chinese doctors experienced more severe psychological symptoms than the general Chinese population [15]. Therefore, it is very important to assess the psychological status of medical workers in community hospitals.

SCL-90 questionnaire is an international, self-report questionnaire to investigate psychological factors. Increasing studies suggest that the SCL-90 questionnaire could be useful in evaluating the patients' psychological states and have made recommendations for psychosocial approaches in clinical practice $[10,16,17]$. There are some researches on the psychological states of doctors and nurses in China. It is reported that the tendency of nurses' job burnout has been neglected in the past 19 years, and the mental health level of nurses in China has declined $[4,18]$. One study once showed the mental health problems 
of nurses were significantly higher than those of doctors, and the main reasons were low occupational levels and adverse workstyles which include shift work, heavy workload and so on [19]. A meta-analysis of Chinese nurses revealed that SOM item-scores and PAR item-scores of nurses were $2.04 \pm 0.55$ and $1.49 \pm 0.53$ in 2016, respectively [18]. Meanwhile, a similar research suggested that SOM item-scores and PAR item-scores in pooled Chinese doctors are $1.57 \pm 0.53$ and $1.50 \pm 0.52$, respectively [15]. There were no normal values of SCL-90 for Chinese medical professionals for now. The available normal values of the Chinese version were derived from a representative state-wide sample with SCL-90R [15], so we didn't manage to compare our results with the SCL-90R norm above. To the best of our knowledge, few studies have addressed the difference on SCL-90 score among health professionals in COVID19 pandemic. In our study, the sum scores of SCL-90 showed no difference among medical workers during the pandemic, but the SOM item-scores and PAR item-scores of nurses were significantly higher.

Somatization is described as a pseudo-clinical condition only mimicking real symptoms of a medical disease, and it mainly reflects physical discomfort, including cardiovascular, gastrointestinal, respiratory and other system discomfort. These disorders often cause significant emotional distress, and management of patients with dysfunctional somatoform disorders is complex and challenging $[20,21]$. During the fighting against COVID-19, nurses had to wear heavy protective clothing and work continuously in the contaminated area every day, which caused nurses to develop symptoms such as back pain, stomach discomfort, muscle aches and dyspnea. All these may be the reasons that nurses had higher SOM item-scores.

Paranoid ideation mainly refers to projective thinking, hostility, suspicion, delusion, passive experience and exaggeration. The higher PAR item-scores, and the more prone to paranoia and extreme individual thinking. As mentioned above, nurses undertook a great deal of work and were in urgent need of recognition and encouragement from society and others. However, due to the nature of work, nurses often had difficulties in gaining social recognition. Therefore, nurses had significantly higher PAR item-scores compared with doctors and technicians.

For further analyzing the causes of psychological symptoms in nurses, we compared the SCL-90 scores judging by sex, profession and education level, and found females and nurses had higher item-scores and were more prone to mental disorders. A series of researches found women showed more symptoms on the depression and somatic complaints, and the increase in the workload of the nursing team had an impact on quality of care and safety for patients $[22,23]$. In our study, $87.1 \%$ of nurses were female and all nurses undertook a high workload, which may be important reasons for mental disorders in nurses. However, no relationship was found between SCL-90 scores and the levels of education.

In order to further clarify the impact of the epidemic situation on nurses' psychological status, we compared the SCL-90 scores of nurses during and after the epidemic and found that the scores of each dimension of SCL-90 in nurses during the pandemic were relatively higher than that in nurses after the pandemic. Considering that there were no differences between the two groups of nurses in terms of 
gender, age and education level, we believed that the emergence of COVID-19 is the main factor leading to psychological problems of nurses.

This study also has some limitations. First, because of the sudden outbreak of epidemic, we were not able to follow up and investigate the mental health status of participants in during-pandemic group. Instead, to observe the changes in the psychological status of the nurses, we managed to conduct a survey on nurses in the same hospital returning from the front line of COVID-19. Second, this study was conducted at a single-center hospital with a limited sample size, there may also be a selection bias. A larger cohort study would help to further define the psychological status of medical workers.

In summary, our study showed that nurses suffered with more psychological symptoms than doctors and technicians when fighting against COVID-19, especially on somatization and paranoid ideation. The emergence of COVID-19 was the main factor leading to psychological problems of nurses.

\section{Declarations}

Funding. None.

Conflicts of interest/Competing interests. All authors declare that they have no conflict of interest.

Ethics approval. The medical ethics committee of Dongxi Lake district people's hospital approved the research protocol.

Consent to participate. All participants gave informed consent.

Consent for publication. All participants gave informed consent.

Availability of data and material. The data that support the findings of this study are available from corresponding author upon reasonable request.

Code availability. Not applicable

Authors' contributions. You-Ping Zhang performed the study and wrote the manuscript. Hai-Tao Yuan designed, supervised the project and wrote the manuscript. Jing Jia assisted in data analysis, and figure preparation. Ya-Qin Song assisted in data acquisition and data analysis. Wei Mi, Yan-Lin Wang and Jia Wang assisted in medical workers' training and data acquisition. Da-Yong Li assisted in study design. Wei Zhu conceived, designed, and supervised the project, and provided financial support.

\section{References}

1. Wu Z, McGoogan JM. Characteristics of and Important Lessons From the Coronavirus Disease 2019 (COVID-19) Outbreak in China: Summary of a Report of 72314 Cases From the Chinese Center for Disease Control and Prevention. JAMA. 2020. doi:10.1001/jama.2020.2648. 
2. Hardy P, Costemale-Lacoste JF, Trichard C, Butlen-Ducuing F, Devouge I, Cerboneschi V, Jacob E, Buferne R, Benyamina A, Cantero A, Gravier V, Ghanem T, Guerin A, Meidinger A, Baleyte JM, Pelissolo A, Corruble E. Comparison of burnout, anxiety and depressive syndromes in hospital psychiatrists and other physicians: Results from the ESTEM study. Psychiatry Res. 2020;284:112662. doi:10.1016/j.psychres.2019.112662.

3. Lalloo D, Demou E, Smedley J, Madan I, Asanati K, Macdonald EB. Current research priorities for UK occupational physicians and occupational health researchers: a modified Delphi study. Occup Environ Med. 2018;75(11):830-6. doi:10.1136/oemed-2018-105114.

4. Ilic IM, Arandjelovic MZ, Jovanovic JM, Nesic MM. Relationships of work-related psychosocial risks, stress, individual factors and burnout - Questionnaire survey among emergency physicians and nurses. Med Pr. 2017;68(2):167-78. doi:10.13075/mp.5893.00516.

5. Xiang YT, Yang Y, Li W, Zhang L, Zhang Q, Cheung T, Ng CH. Timely mental health care for the 2019 novel coronavirus outbreak is urgently needed. Lancet Psychiatry. 2020;7(3):228-9. doi:10.1016/S2215-0366(20)30046-8.

6. Xiao C. A Novel Approach of Consultation on 2019 Novel Coronavirus (COVID-19)-Related Psychological and Mental Problems: Structured Letter Therapy. Psychiatry Investig. 2020;17(2):1756. doi:10.30773/pi.2020.0047.

7. Park SC, Park YC. Mental Health Care Measures in Response to the 2019 Novel Coronavirus Outbreak in Korea. Psychiatry Investig. 2020;17(2):85-6. doi:10.30773/pi.2020.0058.

8. Kang L, Li Y, Hu S, Chen M, Yang C, Yang BX, Wang Y, Hu J, Lai J, Ma X, Chen J, Guan L, Wang G, Ma $\mathrm{H}$, Liu Z. The mental health of medical workers in Wuhan, China dealing with the 2019 novel coronavirus. Lancet Psychiatry. 2020;7(3):e14. doi:10.1016/S2215-0366(20)30047-X.

9. Olsen LR, Mortensen EL, Bech P. The SCL-90 and SCL-90R versions validated by item response models in a Danish community sample. Acta Psychiatr Scand. 2004;110(3):225-9. doi:10.1111/j.1600-0447.2004.00399.x.

10. Numan N. Exploration of adverse psychological symptoms in Yemeni khat users by the Symptoms Checklist-90 (SCL-90). Addiction. 2004;99(1):61-5. doi:10.1111/j.1360-0443.2004.00570.x.

11. Kass F, Charles E, Klein DF, Cohen P. Discordance between the SCL-90 and therapists' psychopathology ratings. Arch Gen Psychiatry. 1983;40(4):389-93. doi:10.1001/archpsyc.1983.01790040043006.

12. Derogatis LR, Rickels K, Rock AF. The SCL-90 and the MMPI: a step in the validation of a new selfreport scale. Br J Psychiatry. 1976;128:280-9. doi:10.1192/bjp.128.3.280.

13. Xu B, Kraemer MUG, Open C-DCG. Open access epidemiological data from the COVID-19 outbreak. Lancet Infect Dis. 2020. doi:10.1016/S1473-3099(20)30119-5.

14. Ji Y, Ma Z, Peppelenbosch MP, Pan Q. Potential association between COVID-19 mortality and healthcare resource availability. Lancet Glob Health. 2020. doi:10.1016/S2214-109X(20)30068-1.

15. Dai Y, Zhang B, Sun H, Li Z, Shen L, Liu Y. Prevalence and Correlates of Psychological Symptoms in Chinese Doctors as Measured with the SCL-90-R: A Meta-Analysis. Res Nurs Health. 2015;38(5):369- 
83. doi:10.1002/nur.21673.

16. Carrozzino D, Morberg BM, Siri C, Pezzoli G, Bech P. Evaluating psychiatric symptoms in Parkinson's Disease by a clinimetric analysis of the Hopkins Symptom Checklist (SCL-90-R). Prog Neuropsychopharmacol Biol Psychiatry. 2018;81:131-7. doi:10.1016/j.pnpbp.2017.10.024.

17. Prinz U, Nutzinger DO, Schulz H, Petermann F, Braukhaus C, Andreas S. Comparative psychometric analyse of the SCL-90-R and its short versions in patients with affective disorders. BMC Psychiatry. 2013;13:104. doi:10.1186/1471-244X-13-104.

18. Xin S, Jiang W, Xin Z. Changes in Chinese nurses' mental health during 1998-2016: A cross-temporal meta-analysis. Stress Health. 2019;35(5):665-74. doi:10.1002/smi.2907.

19. Tong Y, Jiang ZQ, Zhang YX, Jia JL, Lu W, Wang J, Tang HJ, Zhang M, Guo XN, Li T, Jiang HY, Yu WL, Lou JL. [Analyzing the mental health status and its impact factors among female nurses in China]. Zhonghua Lao Dong Wei Sheng Zhi Ye Bing Za Zhi. 2018;36(2):115-8. doi:10.3760/cma.j.issn.10019391.2018.02.009.

20. Salawu FK, Wakil MA, Danburam A. Overview of somatization-diagnosis and management. Niger J Med. 2009;18(4):349-53.

21. Oyama O, Paltoo C, Greengold J. Somatoform disorders. Am Fam Physician. 2007;76(9):1333-8.

22. Beschoner P, Braun M, Schonfeldt-Lecuona C, Freudenmann RW, von Wietersheim J. [Gender aspects in female and male physicians: Occupational and psychosocial stress]. Bundesgesundheitsblatt Gesundheitsforschung Gesundheitsschutz. 2016;59(10):1343-50. doi:10.1007/s00103-016-2431-7.

23. Dekker J, Koelen JA, Peen J, Schoevers RA, Gijsbers-van Wijk C. Gender differences in clinical features of depressed outpatients: preliminary evidence for subtyping of depression? Women Health. 2007;46(4):19-38. doi:10.1300/j013v46n04_02.

\section{Tables}

\section{Table1. Participants characteristics.}

${ }^{*} \mathrm{P}<0.05$ VS. doctors; ${ }^{\#} \mathrm{P}<0.05$ VS. technicians

low education: junior college graduated or below; medium education: bachelor degree; high education: master degree or above. 


\begin{tabular}{|lllll|}
\hline \multicolumn{3}{|c|}{ During-pandemic group } & & After-pandemic group \\
\hline & $\begin{array}{l}\text { Doctors } \\
(\mathrm{n}=36)\end{array}$ & $\begin{array}{l}\text { Technicians } \\
(\mathrm{n}=15)\end{array}$ & $\begin{array}{l}\text { Nurses } \\
(\mathrm{n}=69)\end{array}$ & $\begin{array}{l}\text { Nurses } \\
(\mathrm{n}=34)\end{array}$ \\
\hline Age & $37.50 \pm 7.97$ & $28.67 \pm 6.76$ & $29.67 \pm 5.64^{*}$ & $27.74 \pm 4.65$ \\
\hline Gender & & & & \\
\hline male $(\mathrm{n}, \%)$ & $25(69.4 \%)$ & $10(66.7 \%)$ & $2(12.9 \%)^{*}$ & $1(2.9 \%)$ \\
\hline female $(\mathrm{n}, \%)$ & $11(30.6 \%)$ & $5(33.3 \%)$ & $67(87.1 \%)^{*}$ & $33(97.1 \%)$ \\
\hline Education (n, \%) & & & & \\
\hline low & $1(2.8 \%)$ & $8(53.3 \%)$ & $22(31.9 \%)^{*}$ & $13(38.2 \%)$ \\
\hline medium & $27(75.0 \%)$ & $7(46.7 \%)$ & $47(68.1 \%)^{*}$ & $21(61.8 \%)$ \\
\hline high & $8(22.2 \%)$ & $0(0 \%)$ & $0(0 \%)^{*}$ & $0(0 \%)$ \\
\hline Working hours & $4 \mathrm{~h} /$ day & $6 \mathrm{~h} /$ day & $4 \mathrm{~h} / \mathrm{day}$ & $8 \mathrm{~h} /$ day \\
\hline
\end{tabular}

Table 2. SCL-90 scores of three types of professionals during the pandemic.

${ }^{*} \mathrm{P}<0.05$ VS. doctors; ${ }^{\#} \mathrm{P}<0.05$ VS. technicians

ANX, anxiety; DEP, depression; GSI, global severity index; HOS, hostility; I-S, interpersonal sensitivity; O-C, obsessive-compulsive behavior; PAR, paranoid ideation; PHOB, phobic anxiety; PSY, psychoticism; SOM, somatization. 


\begin{tabular}{|llll|}
\hline & $\begin{array}{l}\text { Doctors } \\
(\mathbf{n = 3 6})\end{array}$ & Technicians $(\mathbf{n = 1 5 )}$ & $\begin{array}{l}\text { Nurses } \\
(\mathbf{n}=69)\end{array}$ \\
\hline SCL-90 sum score & $114.03 \pm 21.19$ & $109.53 \pm 25.45$ & $125.62 \pm 44.08$ \\
\hline SOM & $1.28 \pm 0.31$ & $1.19 \pm 0.27$ & $1.51 \pm 0.54^{* \#}$ \\
\hline O-C & $1.46 \pm 0.34$ & $1.33 \pm 0.47$ & $1.64 \pm 0.66$ \\
\hline I-S & $1.18 \pm 0.25$ & $1.23 \pm 0.35$ & $1.30 \pm 0.47$ \\
\hline DEP & $1.28 \pm 0.33$ & $1.20 \pm 0.34$ & $1.36 \pm 0.54$ \\
\hline ANX & $1.26 \pm 0.33$ & $1.27 \pm 0.36$ & $1.44 \pm 0.56$ \\
\hline HOS & $1.28 \pm 0.45$ & $1.17 \pm 0.23$ & $1.37 \pm 0.64$ \\
\hline PHOB & $1.17 \pm 0.31$ & $1.17 \pm 0.26$ & $1.28 \pm 0.45$ \\
\hline PAR & $1.09 \pm 0.16$ & $1.11 \pm 0.16$ & $1.23 \pm 0.41^{*}$ \\
\hline PSY & $1.11 \pm 0.17$ & $1.11 \pm 0.22$ & $1.20 \pm 0.36$ \\
\hline GSI & $1.27 \pm 0.24$ & $1.22 \pm 0.28$ & $1.40 \pm 0.49$ \\
\hline
\end{tabular}

Table 3. SOM and PAR item-scores of three types of professionals during the pandemic.

${ }^{*} \mathrm{P}<0.05$ VS. doctors. SOM, somatization; PAR, paranoid ideation. 


\begin{tabular}{|c|c|c|c|c|}
\hline & items & Doctors & Technicians & Nurses \\
\hline \multirow[t]{12}{*}{ SOM } & 1 & $1.67 \pm 0.96$ & $1.33 \pm 0.62$ & $1.65 \pm 0.76$ \\
\hline & 4 & $1.17 \pm 0.38$ & $1.27 \pm 0.46$ & $1.38 \pm 0.67$ \\
\hline & 12 & $1.31 \pm 0.67$ & $1.07 \pm 0.26$ & $1.28 \pm 0.51$ \\
\hline & 27 & $1.75 \pm 0.94$ & $1.53 \pm 0.92$ & $2.30 \pm 1.15^{* \#}$ \\
\hline & 40 & $1.25 \pm 0.60$ & $1.27 \pm 0.70$ & $1.68 \pm 0.85$ *\# \\
\hline & 42 & $1.36 \pm 0.80$ & $1.27 \pm 0.46$ & $1.78 \pm 0.94$ *\# \\
\hline & 48 & $1.14 \pm 0.35$ & $1.07 \pm 0.26$ & $1.39 \pm 0.71$ * \\
\hline & 49 & $1.06 \pm 0.23$ & $1.07 \pm 0.26$ & $1.28 \pm 0.64$ * \\
\hline & 52 & $1.08 \pm 0.37$ & $1.00 \pm 0.00$ & $1.30 \pm 0.58$ *\# \\
\hline & 53 & $1.25 \pm 0.44$ & $1.33 \pm 0.82$ & $1.33 \pm 0.63$ \\
\hline & 56 & $1.17 \pm 0.45$ & $1.07 \pm 0.26$ & $1.43 \pm 0.79$ \\
\hline & 58 & $1.14 \pm 0.42$ & $1.00 \pm 0.00$ & $1.36 \pm 0.79$ \\
\hline \multirow[t]{6}{*}{ PAR } & 8 & $1.33 \pm 0.63$ & $1.07 \pm 0.26$ & $1.41 \pm 0.67$ \\
\hline & 18 & $1.06 \pm 0.23$ & $1.27 \pm 0.59$ & $1.30 \pm 0.55$ * \\
\hline & 43 & $1.00 \pm 0.00$ & $1.00 \pm 0.00$ & $1.07 \pm 0.26$ \\
\hline & 68 & $1.08 \pm 0.28$ & $1.27 \pm 0.46$ & $1.25 \pm 0.76$ \\
\hline & 76 & $1.08 \pm 0.28$ & $1.07 \pm 0.26$ & $1.30 \pm 0.60$ * \\
\hline & 83 & $1.00 \pm 0.00$ & $1.00 \pm 0.00$ & $1.04 \pm 0.21$ \\
\hline
\end{tabular}

Table 4. The SCL-90 scores of nurses during the pandemic and after the pandemic.

${ }^{*} \mathrm{P}<0.05$

ANX, anxiety; DEP, depression; GSI, global severity index; HOS, hostility; I-S, interpersonal sensitivity; O-C, obsessive-compulsive behavior; PAR, paranoid ideation; PHOB, phobic anxiety; PSY, psychoticism; SOM, somatization. 


\begin{tabular}{|lll|}
\hline & $\begin{array}{l}\text { Nurses during pandemic } \\
(\mathbf{n}=69)\end{array}$ & $\begin{array}{l}\text { Nurses after pandemic } \\
(\mathbf{n}=\mathbf{3 4})\end{array}$ \\
\hline SCL-90 sum score & $125.62 \pm 44.08$ & $107.24 \pm 15.74^{\star}$ \\
\hline SOM & $1.51 \pm 0.54$ & $1.21 \pm 0.24^{\star}$ \\
\hline O-C & $1.64 \pm 0.66$ & $1.44 \pm 0.33^{\star}$ \\
\hline I-S & $1.30 \pm 0.47$ & $1.12 \pm 0.24^{\star}$ \\
\hline DEP & $1.36 \pm 0.54$ & $1.17 \pm 0.17^{\star}$ \\
\hline ANX & $1.44 \pm 0.56$ & $1.19 \pm 0.18^{\star}$ \\
\hline HOS & $1.37 \pm 0.64$ & $1.24 \pm 0.27$ \\
\hline PHOB & $1.28 \pm 0.45$ & $1.12 \pm 0.18^{\star}$ \\
\hline PAR & $1.23 \pm 0.41$ & $1.12 \pm 0.20$ \\
\hline PSY & $1.20 \pm 0.36$ & $1.06 \pm 0.16^{\star}$ \\
\hline GSI & $1.40 \pm 0.49$ & $1.19 \pm 0.17^{\star}$ \\
\hline
\end{tabular}

Figures 
a

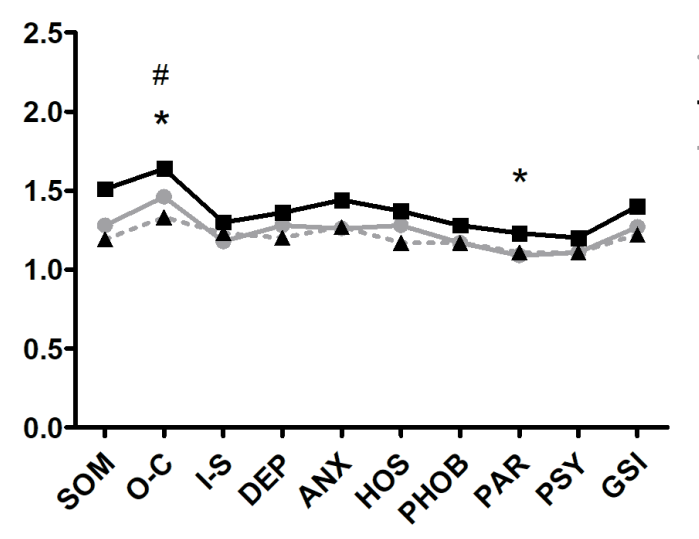

C

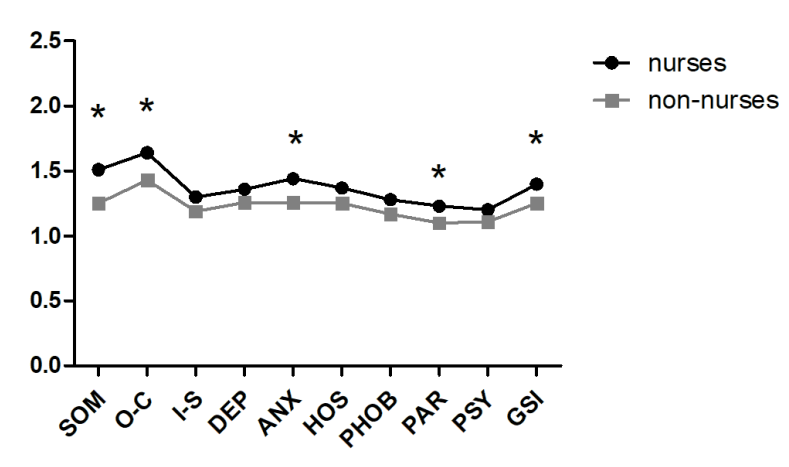

b

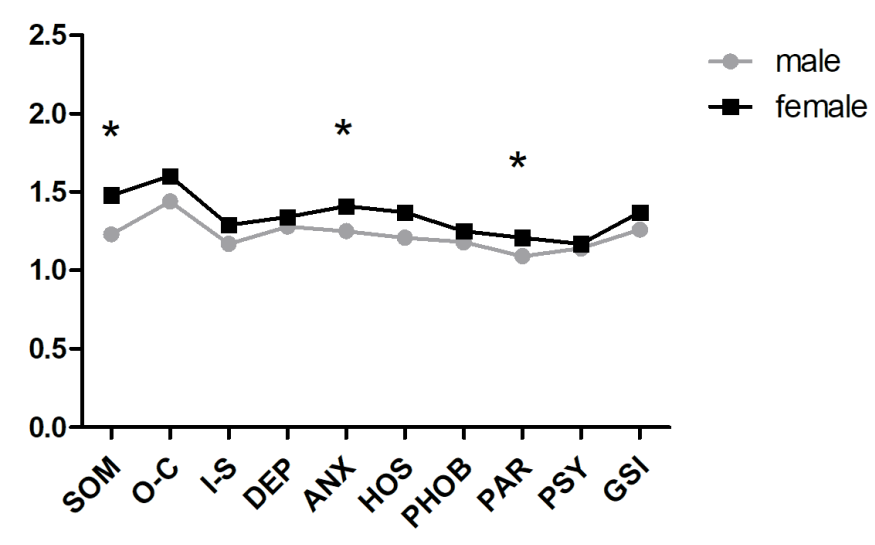

d

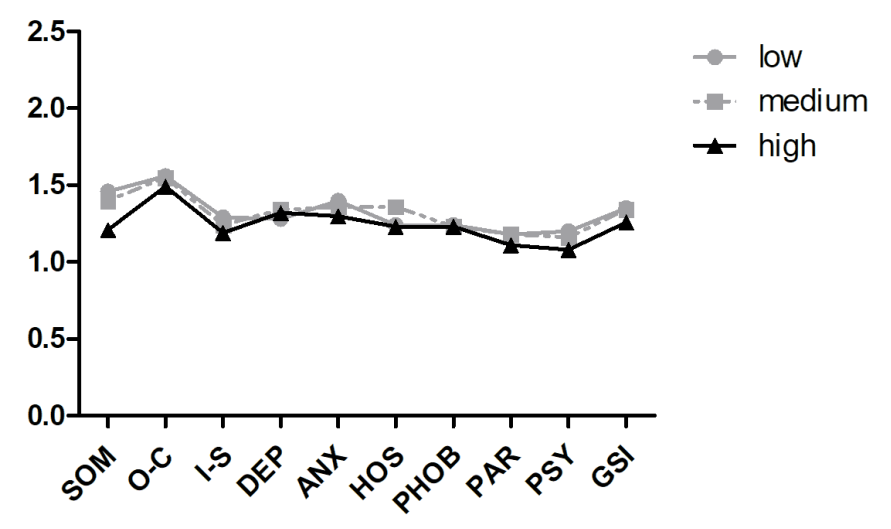

\section{Figure 1}

Influence of gender, profession and education level on the SCL-90 score during the pandemic. (a) The SCL-90 scores among doctors, nurses and technicians. ${ }^{*} \mathrm{P}<0.05$ (nurses VS. doctors); $\# \mathrm{P}<0.05$ (nurses VS. technicians). (b) The SCL-90 scores between females and males. ${ }^{*} \mathrm{P}<0.05$. (c) the SCL-90 scores between nurses and non-nurses. ${ }^{*} P<0.05$. (d) The SCL-90 scores among different education levels. 


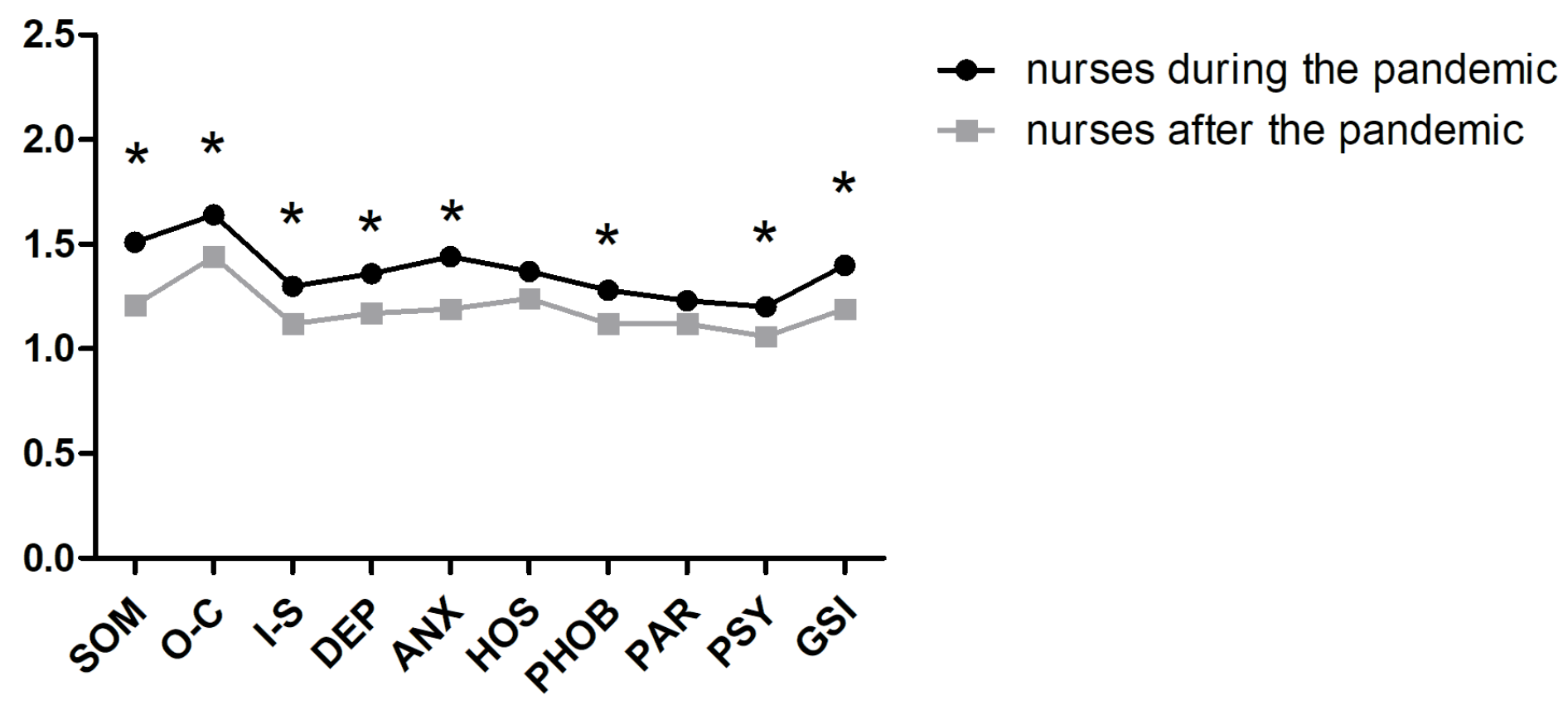

Figure 2

The scores of nurses during the pandemic and after the pandemic. ${ }^{*} \mathrm{P}<0.05$ 